\title{
Particle therapy in non-small cell lung cancer
}

\author{
Zhongxing Liao ${ }^{1}$, Charles B. Simone II $^{2}$ \\ ${ }^{1}$ Department of Radiation Oncology, The University of Texas MD Anderson Cancer Center, Houston, TX, USA; ${ }^{2}$ Department of Radiation \\ Oncology, University of Maryland Medical Center, Baltimore, MD, USA \\ Contributions: (I) Conception and design: All authors; (II) Administrative support: CF Wogan; (III) Provision of study materials or patients: All \\ authors; (IV) Collection and assembly of data: All authors; (V) Data analysis and interpretation: All authors; (VI) Manuscript writing: All authors; (VII) \\ Final approval of manuscript: All authors. \\ Correspondence to: Zhongxing Liao, MD. Department of Radiation Oncology, Unit 1422, The University of Texas MD Anderson Cancer Center, 1400 \\ Pressler Street, Houston, TX 77030, USA. Email: zliao@mdanderson.org.
}

\begin{abstract}
The finite range of proton beams in tissues offers unique dosimetric advantages that theoretically allow the dose to the target to be escalated while minimizing exposure of surrounding tissues and thereby minimizing radiation-induced toxicity. These theoretical advantages have led to widespread adoption of proton therapy around the world for a wide variety of tumors at different anatomic sites. Many treatmentplanning comparisons have shown that proton therapy has substantial dosimetric advantages over conventional photon (X-ray) radiation therapy. However, given the typically significant difference in cost between proton therapy versus conventional photon therapy, strong evidence of proton therapy's clinical benefits in terms of toxicity and survival is warranted. Some findings from retrospective studies, singlearm prospective studies, and a very few randomized clinical trials comparing these modalities are beginning to emerge. In this review, we examine the available data on proton therapy for (non-small cell lung cancer NSCLC). We begin by discussing the unique challenges involved in treating moving targets with significant tissue heterogeneity and the technologic efforts underway to overcome these challenges. We then discuss the rationale for minimizing normal tissue toxicity, particularly pulmonary, cardiac, and hematologic toxicity, within the context of previously unsuccessful attempts at dose escalation for lung cancer. Finally, we explore strategies for accelerating the development of trials aimed at measuring meaningful clinical endpoints and for maximizing the value of proton therapy by personalizing its use for individual patients.
\end{abstract}

Keywords: Proton beam therapy; lung cancer; passively scatted proton therapy; intensity modulated proton therapy

Submitted Dec 12, 2017. Accepted for publication Apr 16, 2018.

doi: $10.21037 /$ tlcr.2018.04.11

View this article at: http://dx.doi.org/10.21037/tlcr.2018.04.11

\section{Introduction}

Thoracic malignancies such as non-small cell lung cancer (NSCLC) and esophageal cancer are complex and challenging to treat. They are often diagnosed at locally advanced stages and are not amenable to surgical resection. In such cases, radiation therapy, given with either concurrent or sequential chemotherapy, is often the treatment of choice. Unfortunately, most patients with locally advanced lung or esophageal cancer die of the disease; the median survival times even with treatment are only 16-28 months, and local recurrence accounts for $40-50 \%$ of failures. Although radiation dose escalation has been tested as a strategy to improve tumor control and patient survival, recent phase III randomized studies investigating dose intensification of thoracic irradiation showed that higher radiation doses conferred no benefit to patients with locally advanced NSCLC or esophageal cancer $(1,2)$. No differences were found in local control between the standard-dose and high-dose arms, and the higher-dose 
radiation had a detrimental effect on patient survival.

The fact that the cancer-related death rates for the standard-dose and high-dose groups in both studies were similar suggests that the higher death rate in the high-dose group was from non-cancer-related reasons, specifically treatment-related toxicity. The results of the Radiation Therapy Oncology Group (RTOG) 0617 trial showed that the radiation dose to the heart was an independent predictor of survival, confirming that exposing larger portions of the heart to higher thoracic radiation doses contributed to the higher death rates in the high-dose arm (1). Several current reports have also linked therapy-induced lymphopenia during chemoradiation with poor survival for many types of tumors, including lung cancer, esophageal cancer, head and neck cancer, gastrointestinal cancer, and cervical cancer (3-6).

The relevant organs at risk in the treatment of thoracic malignancies are the esophagus, lungs, heart, and bone marrow; other important structures or tissues include the brachial plexus, skin, spinal cord, and chest wall. In principle, the most effective strategy to reduce toxicity would be to reduce unnecessary irradiation of organs at risk by using advanced technologies, one example of which is proton beam therapy. Proton therapy offers substantial potential advantages over conventional photon therapy because of the unique depth-dose characteristics of protons, which can be exploited to reduce irradiation of normal tissues proximal and distal to the target volume so as to allow escalation of tumor doses while simultaneously sparing greater amounts of normal tissues; the expectation is that these effects would improve local tumor control and survival as well as reducing treatment-related toxicity and improving quality of life.

However, particle therapy (including proton therapy) is significantly more expensive than even the best available photon technology to date, and evidence demonstrating clinical benefit after proton therapy is increasingly demanded to justify the higher financial burden on the healthcare delivery system. Despite the high capital costs associated with charged particle therapy and the lack of level I evidence of clinical benefit from direct comparisons, the increasing demand for improved technology in cancer treatment, particularly proton therapy, is evidenced by the numbers of facilities being built worldwide. Currently, 76 particle therapy centers are in operation, 25 of which are proton centers in the United States, and many more are being planned (Particle Therapy Cooperative Group, https://www.ptcog.ch/index.php/). By 2015, more than 154,000 patients worldwide had been treated with charged particle therapy (https://www.ptcog.ch/index.php/). Parallel with this increase in the numbers of facilities and the clinical use of particle therapy is the accumulation of knowledge about the physical uncertainties of particle therapy and methods of counteracting these uncertainties to ensure accurate planning and precise delivery of particle therapy.

In this review, we summarize the rationale for and challenges of using charged particles to treat thoracic cancers; we review the clinical experience to date on the use of proton therapy for locally advanced lung cancer and esophageal cancer; and we discuss future directions for use of proton therapy.

\section{Dosimetry and radiobiology of charged particle therapy}

The radiobiology and dosimetric characteristics of charged particle therapy have been reviewed in depth elsewhere (7). Briefly, charged particle radiotherapy involves the use of charged particles such as protons or carbon ions to treat cancer. The depth dose characteristics of charged particles are well understood and described elsewhere (8). When a "fast" charged particle moves through matter, it interacts with the electrons within atoms and causes ionizations, which deposit energy and dose along its path. The energy loss per unit path length is relatively constant until it reaches a peak (the so-called Bragg peak), where energy deposition occurs at a depth that is a function of the energy and nature of the charged particle. Beyond that Bragg peak, very little dose remains. In passively scattering proton therapy (PSPT), the Bragg peak is spread both longitudinally and laterally to create a spread-out Bragg peak (SOBP), which provides a uniform dose to cover the entire volume of a target. Conformal coverage of the tumor is achieved by using range modulation wheels, compensators, and beam apertures. Pencil beam scanning proton therapy, on the other hand, uses magnetic scanning of thin beamlets of protons of a sequence of energies, delivered from different directions, to produce the desired pattern of dose distribution. The tumor is "scanned" layer by layer, with one layer per energy, until the entire target is covered. This technique provides greater flexibility and control for ideal dose distributions and allows delivery of intensity-modulated proton therapy (IMPT), to date the most advanced form of proton therapy (7). Many treatment-planning comparison studies have demonstrated dosimetric advantages of IMPT over intensity-modulated photon radiation therapy (IMRT) $(9,10)$.

The biological interaction of ionizing radiation with 
matter (i.e., tissues) is related to the amount of energy transferred to the matter over a specified path length [known as linear energy transfer (LET)]. For particles such as protons and helium, the LET is thought to be nearly equivalent to that of photons and, therefore, the relative biological effectiveness (RBE) is also nearly equivalent (the $\mathrm{RBE}$ for protons:photons is approximately 1.1$)(11,12)$. For heavier charged particles such as carbon ions, the density of ionization is greater at the end of their range, which causes greater damage to the DNA within cells at the end of that range and results in carbon ions having a higher RBE (1.5-3). However, it is becoming increasingly evident that $\mathrm{RBE}$ is a complex, variable function of radiation dose per fraction, total dose, LET, cell and tissue type, choice of endpoint, and other factors $(13,14)$. Thus, the RBE may be less than 1.1 at the entrance, may increase with depth, and may be highest at the distal edge of the beam.

These two physical properties of protons, i.e., having a finite range in tissue and having a higher RBE at the distal edge of the beam, make proton therapy both appealing and potentially problematic. Proton therapy is exquisitely sensitive to changes in tumor position and density and to differences in tissue composition; this sensitivity is particularly problematic for thoracic tumors, because the tumors move with lung ventilation and diaphragm motion, and because the tissues along the beam path are quite heterogeneous in structure and density. In PSPT, extreme care must be taken to consider the need to compensate for tumor motion, changes in lung density due to respiration, and uncertainties in proton range with regard to respirationinduced tumor motion and lung density changes (Figure 1). These variables should be assessed separately for each beam direction, and some amount of dosimetric uncertainty should be built into the planning of each beam (15). Although lung motion and density uncertainties can be accounted for during the treatment planning process by adding generous internal and smearing margins, practical issues regarding inconsistencies in patient setup and positioning and changes in tumor volume between treatment sessions must also be accounted for during the course of treatment (16).

In IMPT, conformity of the proximal and lateral field is achieved by limiting the position of the spots to within the target region only. Dynamic apertures that can change shape layer by layer are being developed to address the issues of large spots appearing in pencil beam scanning proton therapy. In treatment planning, the position and intensities for a matrix of spots within the target volume for each scanned beam are calculated automatically by the treatment planning system to achieve the desired dose distribution. The IMPT dose distribution is more sensitive to uncertainties in set-up and motion than is the PSPT dose distribution. To address this heightened sensitivity, "robust optimization" techniques that simultaneously consider multiple uncertainty scenarios and optimize intensities in the face of all those scenarios are being actively investigated $(17,18)$.

\section{Clinical outcomes after proton therapy for NSCLC}

Despite the technical challenges associated with using proton therapy for lung cancer, where the heterogeneity of treated tissues leads to significant uncertainties in dose, the number of new proton centers has surged worldwide, and more than 154,000 patients with a variety of tumor types had been treated by 2015 . However, published results have been mostly from retrospective, single-institution series, national databases, or single-arm prospective studies. Clinical evidence from prospective randomized trials of proton therapy is just now emerging.

\section{Early-stage NSCLC}

Representative studies of particle therapy for early-stage NSCLC published since 2010 are shown in Table 1 (19-25). One prospective phase II dose-escalation study (21) reported a dose-dependent improvement in overall survival. In that study, 111 patients with early-stage NSCLC (47 with T1, 64 with T2, $40 \%$ central and $60 \%$ peripheral) were treated with the tumor dose prescribed to the center of the target volume and escalated sequentially to reach 51 and $60 \mathrm{~Gy}$ in 10 fractions. An interim analysis showed that local control was insufficient with 60 Gy and the protocol was amended to increase the dose to 70 Gy in 10 fractions during the final phase of the trial. No clinically significant radiation pneumonitis was observed. With a minimum follow-up time for all subjects of 3 years (median, 48 months), a dosedependent improvement in survival was observed, in that the 4-year overall survival rates were $18 \%$ after $51 \mathrm{~Gy}$, $32 \%$ after $60 \mathrm{~Gy}$, and $51 \%$ after 70 Gy. For patients with peripheral $\mathrm{T} 1$ tumors, at 4 years the local control rate was $96 \%$, the disease-specific survival rate was $88 \%$, and the overall survival rate was $60 \%$, results comparable with those after photon stereotactic ablative radiation therapy (SABR) (26). Patients with T2 tumors showed a trend toward improved local control and survival at the 70-Gy 

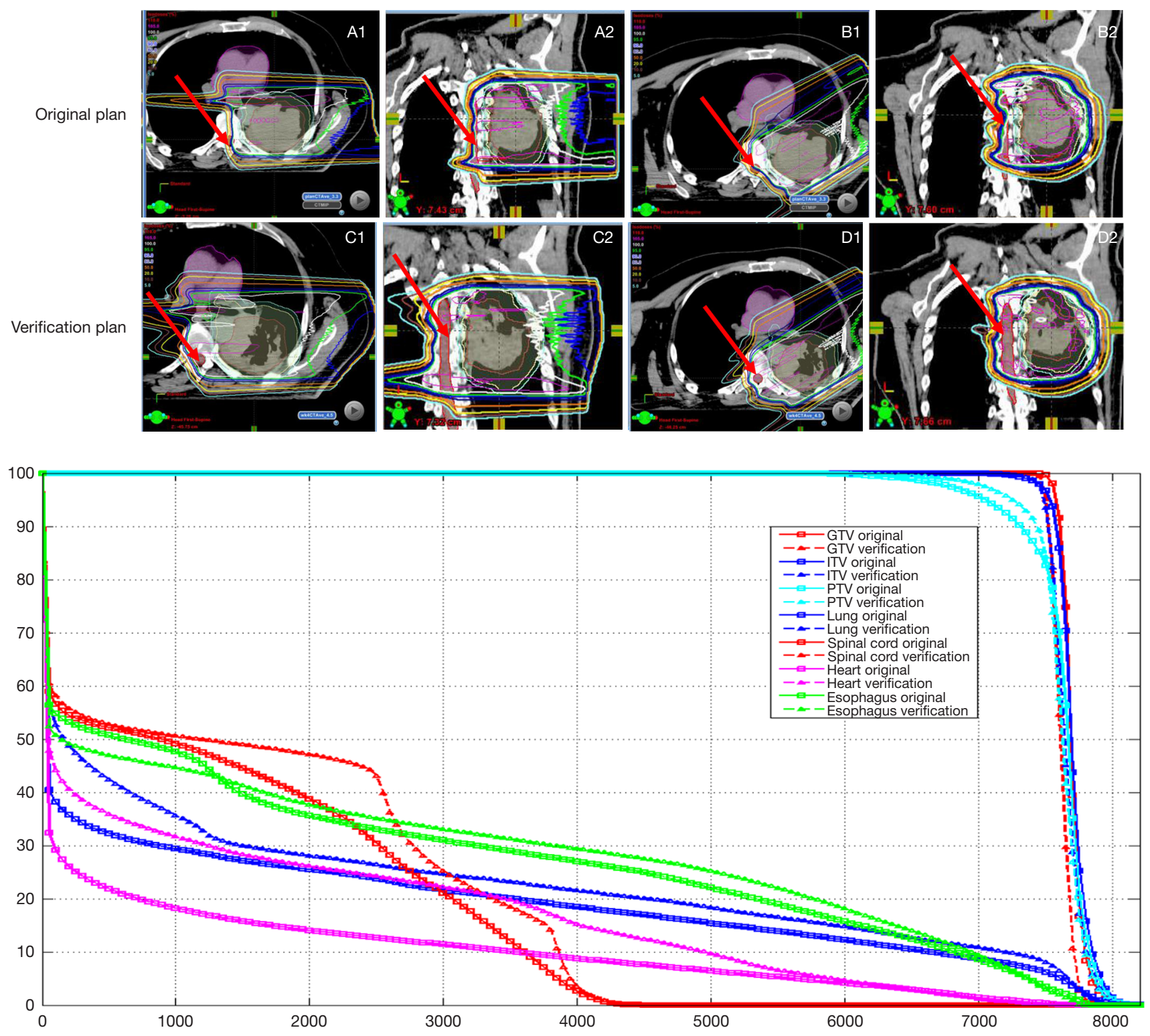

Figure 1 Proton dose distribution is vulnerable to changes in anatomy. First row, dose distribution of the initial plan for 4-field passively scattering proton therapy (PSPT) in axial and sagittal views from the (A) left lateral (A1, axial view, A2, coronal view) and (B) left anterior oblique fields (B1, axial view, B2, coronal view). Second row, verification plan in week 4 (C1, lateral field, axial view, C2, lateral field, coronal view) (D1, left anterior oblique field, axial view, D2, left anterior oblique field, coronal view). Note the overshoot of protons to the spinal cord due to tumor cavitation. Third row, dose-volume histogram of initial (square) and verification (triangle) plan showing increased dose to the heart, lung, esophagus, and the spinal cord. Red arrows indicate the dose difference in spinal cord due to tumor shrinkage.

dose level. Tumor size was the only factor that predicted increased local recurrence and decreased survival, whereas central versus peripheral location did not correlate with any outcome measures. Those authors have adopted the 70-Gy regimen as standard therapy for T1 tumors (21). However, it was not clear from the report whether the radiation dose was corrected for RBE, or what the dose coverage was for the planning target volume because the radiation dose was 
Table 1 Reports of clinical outcomes after particle therapy for early-stage non-small cell lung cancer since 2010

\begin{tabular}{|c|c|c|c|c|c|c|}
\hline Reference & $\begin{array}{l}\text { Disease } \\
\text { stage (No. } \\
\text { of tumors) }\end{array}$ & $\begin{array}{l}\text { Particle dose and No. of } \\
\text { fractions }\end{array}$ & Overall survival rate & Local control rate & $\begin{array}{l}\text { Progression-free } \\
\text { survival rate }\end{array}$ & Comments \\
\hline $\begin{array}{l}\text { Iwata et al., } \\
2010 \text { (19) }\end{array}$ & $\begin{array}{l}\text { IA }(n=42) \\
\text { IB }(n=38)\end{array}$ & $\begin{array}{l}\text { Protons: } 60 \text { CGE in } 10 \\
\text { fractions }(n=37), 80 \text { CGE in } 20 \\
\text { fractions }(n=20) \text {. Carbon ions: } \\
52.8 \text { CGE in } 4 \text { fractions }(n=23)\end{array}$ & $3-y r$ 75\% & $3-y r 82 \%$ & Not reported & $\begin{array}{l}\text { Dose prescribed to center of } \\
\text { tumor; no difference in any } \\
\text { measured clinical outcome } \\
\text { between CIT vs. PBT }\end{array}$ \\
\hline $\begin{array}{l}\text { Bush et al., } \\
2013 \text { (21) }\end{array}$ & $\begin{array}{l}\text { IA }(n=47) \\
\text { IB }(n=64)\end{array}$ & $\begin{array}{l}51 \text { Gy in } 10 \text { fractions }(n=29) \text {, } \\
60 \text { Gy in } 10 \text { fractions }(n=56) \text {, } \\
70 \text { Gy in } 10 \text { fractions }(n=26)\end{array}$ & $\begin{array}{l}\text { 4-yr: } 51 \text { Gy, 18\%; } 60 \\
\text { Gy, 32\%; } 70 \text { Gy, } 51 \%\end{array}$ & $\begin{array}{l}\text { 4-yr: T1 } 70 \text { Gy, } \\
\text { 91\%; T1 } 60 \text { Gy, } \\
86 \%\end{array}$ & Not reported & $\begin{array}{l}\text { Radiation dose not corrected for } \\
\text { RBE; dose prescribed to center } \\
\text { of the internal target volume; } \\
95 \% \text { coverage of target }\end{array}$ \\
\hline $\begin{array}{l}\text { Fujii et al., } \\
2013 \text { (23) }\end{array}$ & $\begin{array}{l}\text { IA }(n=62) \\
\text { IB }(n=49)\end{array}$ & $\begin{array}{l}\text { Proton }(n=70): 60 \text { GyE in } 10 \\
\text { fractions }(n=35), 80 \text { GyE in } 20 \\
\text { fractions }(n=16), 66 \text { GyE in } 10 \\
\text { fractions }(n=10), 52.8 \text { GyE in } 4 \\
\text { fractions }(n=7), 70.2 \text { GyE in } 26 \\
\text { fractions }(n=2) \text {. Carbon }(n=41) \text { : } \\
52.8 \text { GyE in } 4 \text { fractions }(n=30) \text {, } \\
66 \text { GyE in } 10 \text { fractions }(n=7), \\
68.4 \text { GyE in } 9 \text { fractions }(n=3), \\
70.2 \text { GyE in } 26 \text { fractions }(n=1)\end{array}$ & $\begin{array}{l}\text { Protons: 3-yr } 72 \% \\
\text { [61-83\%,T1 tumor } \\
\text { (IA) } 73 \%, \text { T2a tumor } \\
\text { (IB) } 70 \%] \text {; 3-yr } \\
\text { progression-free } \\
\text { survival, } 44 \% \text { (32- } \\
56 \%, \text { IA: } 52 \%, \text { IB: } \\
37 \% \text { ); 3-yr: } 80 \text { GyE in } \\
20 \text { fractions } 87 \%, 60 \\
\text { GyE in } 10 \text { fractions } \\
57 \%, 66 \text { GyE in } \\
10 \text { fractions } 87 \% \text {. } \\
\text { Carbon: 3-yr } 76 \% \\
\text { (62-90\%; IA } 84 \%, I B \\
64 \% \text { ); 3-yr: 52.8 GyE } \\
\text { in } 4 \text { fractions } 77 \%, 66 \\
\text { GyE in } 10 \text { fractions } \\
68 \%\end{array}$ & $\begin{array}{l}\text { Proton: 3-yr } 81 \% \\
\text { (70-91\%, IA } \\
90 \%, \text { IB } 72 \%) ; \\
78 \% \text { after } 80 \text { GyE } \\
\text { in } 20 \text { fractions, } \\
80 \% \text { after } 60 \text { GyE } \\
\text { in } 10 \text { fractions, } \\
87 \% \text { after } 66 \text { GyE } \\
\text { in } 10 \text { fractions. } \\
\text { Carbon: 3-yr } 78 \% \\
\text { (64-92\%; IA 80\%, } \\
\text { IB } 73 \%) ; 76 \% \\
\text { after } 52.8 \text { GyE in } \\
4 \text { fractions, } 55 \% \\
\text { after } 66 \text { GyE in } 10 \\
\text { fractions }\end{array}$ & $\begin{array}{l}\text { Protons: 3-yr } \\
\text { PFS rates were } \\
44 \% \text { (32-56\%, } \\
\text { IA 52\%, IB 37\%). } \\
\text { Carbon: 3-yr } \\
\text { PFS rates were } \\
53 \% \text { (37-68\%, } \\
\text { IA 54\%, IB 51\%) }\end{array}$ & $\begin{array}{l}\text { No difference between carbon } \\
\text { and protons in tumor outcomes } \\
\text { or long-term toxicity; Pts with } \\
\text { adenocarcinoma had higher } \\
\text { 3-year OS rate (82\%) than did } \\
\text { pts with SCC }(53 \%)\end{array}$ \\
\hline $\begin{array}{l}\text { Kanemoto } \\
\text { et al., } 2014 \\
(24)\end{array}$ & $\begin{array}{l}\text { IA }(n=59) \\
\text { IB }(n=21)\end{array}$ & $\begin{array}{l}66 \text { CGE in } 10-12 \text { fractions for } \\
\text { peripheral }(n=59), 72.6 \text { CGE in } \\
22 \text { fractions for central }(n=21)\end{array}$ & $5-y r 65.8 \%$ & $5-y r 81.8 \%$ & $5-y r 52.5 \%$ & \\
\hline $\begin{array}{l}\text { Makita et al., } \\
2015(25)\end{array}$ & $\begin{array}{l}\text { IA }(n=43) \\
\text { IB }(n=13)\end{array}$ & $\begin{array}{l}66 \text { CGE in } 10 \text { fractions for } \\
\text { peripheral tumors ( } n=32), 80 \\
\text { CGE in } 25 \text { fractions for central } \\
\text { tumors }(n=24)\end{array}$ & $3-y r 81.3 \%$ & $3-y r$ 96\% & $3-y r 73.4 \%$ & $\begin{array}{l}\text { Dose prescribed to } 90 \% \text { PTV; } \\
\text { SUVmax }<5 \text { vs. } \geq 5 \text { was the only } \\
\text { predictor of OS and PFS }\end{array}$ \\
\hline
\end{tabular}

CIT, carbon ion therapy; PBT, proton beam therapy; RBE, relative biological effectiveness; CGE, cobalt-Gray equivalents; GyE, Gray equivalents; pts, patients; OS, overall survival; SCC, squamous cell carcinoma; PTV, planning target volume; SUVmax, maximum standardized uptake value; PFS, progression-free survival. 
prescribed to the center of the tumor.

Another prospective trial reported by Makita and colleagues (25) involved 56 patients with clinically diagnosed, stage I NSCLC (43 T1, 13 T2). Patients with peripherally located tumors were given $66 \mathrm{GY}$ (RBE) in 10 fractions, whereas patients with centrally located tumors $(\mathrm{n}=24)$ were given $80 \mathrm{GY}$ (RBE) in 25 fractions between January 2009 and May 2012. The radiation dose was prescribed to cover $90 \%$ of the planning target volume, which was defined as the gross tumor volume surrounded with a $10-\mathrm{mm}$ margin. At 3 years, the overall survival rate was $81.3 \%$, the progression-free survival rate was $73.4 \%$, and the local control rate was $96.0 \%$. No significant differences in outcomes were found between the two dose regimens. Late grade 2 pulmonary toxicity occurred in nine patients $(13.4 \%)$, and one patient $(1.5 \%)$ had late grade 3 pulmonary toxicity; no grade 4 or 5 toxicity was noted. Only the maximum standardized uptake value $\left(\mathrm{SUV}_{\max }\right)$ on positron emission tomography $(<5 v s . \geq 5)$ predicted overall and progression-free survival (25). A more recent phase I study of 25 patients with stage I NSCLC treated with proton beam therapy to 60 Gy in 8 fractions has been completed and the results are currently in press (27).

In terms of carbon and proton therapy for early stage NSCLC, Iwata et al. (19) reported results from a series of single-institution protocols that included 80 patients with stage I NSCLC treated with either proton therapy $(n=57)$ or carbon-ion therapy $(n=23)$. In the first proton-therapy protocol, protons were given to $80 \mathrm{GY}$ (RBE) in 20 fractions, and in the second proton-therapy protocol, protons were given to $60 \mathrm{GY}$ (RBE) in 10 fractions. For the carbon-ion therapy, $52.8 \mathrm{GY}$ (RBE) was given in 4 fractions. After promising preliminary results were achieved with the first protocol, the investigators began to use the second proton-therapy protocol to shorten the overall treatment time. Use of carbon-ion therapy began in 2005; thereafter, both proton and carbon-ion therapy plans were created for each patient, and the superior of the two plans was used to deliver the therapy. The median follow-up time for living patients in the carbon-ion study was 35.5 months. For all 80 patients, the 3 -year rates of overall survival were $75 \%$ (74\% IA, 76\% IB); cause-specific survival, 86\% (84\% IA, 88\% IB); and local control 82\% (87\% IA, 77\% IB). No significant differences in treatment results were noted among the 3 protocols or between carbon therapy $v s$. proton therapy. The same investigators also analyzed outcomes in terms of tumor size for 70 patients with early-stage NSCLC (47 T2a, 23 T2b) treated from April 2003 through December 2009 with proton therapy $(n=43)$ or carbon-ion therapy $(\mathrm{n}=27)$ on prospective institutional protocols (22). The total dose delivered to the center of the tumor was $60 \mathrm{GY}$ (RBE) in 10 fractions (20 patients), $52.8 \mathrm{GY}$ (RBE) in 4 fractions (16 patients), $66 \mathrm{~Gy}(\mathrm{RBE}$ in 10 fractions (16 patients), $80 \mathrm{GY}$ (RBE) in 20 fractions (14 patients), or other (4 patients). The median follow-up period was 51 months for patients alive at the time of analysis. For the entire group of 70 patients, the 4-year rate of overall survival was 58\% (53\% T2a, 67\% T2b); local control, 75\% (70\% T2a, 84\% T2b), and progressionfree survival $46 \%$ (43\% T2a, 52\% T2b). The 4-year regional recurrence rate was $17 \%$. Grade 3 pulmonary toxicity was observed in only two patients. Notably, pairs of carbon-ion and proton therapy plans were developed for each patient, and the therapy with the better plan was delivered. No differences were found between carbon-ion therapy and proton therapy in all measured clinical outcomes. Adjuvant chemotherapy was recommended as a reasonable option, whenever possible, to further improve treatment outcome (22). Results from another retrospective study comparing carbon and proton therapy were consistent with those from Iwata et al. in that no difference was found between the two modalities in any measured clinical outcomes (23). A recent meta-analysis of overall survival after SABR versus hypofractionated proton therapy showed no difference in multivariate analysis, even though hypofractionated proton therapy was associated with improved overall survival in univariate analysis (28).

Image-guided SABR [also known as stereotactic body radiation therapy (SBRT)], has become the treatment of choice for patients with medically or surgically inoperable disease and is also an excellent alternative for patients who are candidates for surgery but choose to undergo nonsurgical therapy $(29,30)$. For patients with peripherally located stage IA NSCLC, image-guided SABR has produced excellent local control rates (range $80 \%$ to $97.6 \%$ ) and survival rates of about $55 \%$ with minimal toxicity (30,31). A biologically effective dose (BED) of $105 \mathrm{~Gy}_{10}$ or higher has been associated with better overall survival (median survival times 28 months with BED $\geq 105$ Gy $v s$. 22 months with BED <105 Gy) (32). Among patients with larger tumors, a BED of 150 Gy was noted to be associated with better tumor control and survival (33).

Although peripherally located lung lesions can be treated to high BEDs, high-BED treatment to centrally located lesions can result in considerable long-term toxicity because of the proximity of critical structures such as the bronchi, major vessels, heart, spinal cord, esophagus, and trachea (26). With image-guided interventions and other improvements in technology, proton therapy may allow the doses to be 
Table 2 Pneumonitis and overall survival after concurrent chemoradiation therapy for locally advanced non-small cell lung cancer

\begin{tabular}{|c|c|c|c|c|c|c|}
\hline Reference & $\begin{array}{l}\text { Total No. } \\
\text { of patients }\end{array}$ & Disease stage & \multicolumn{2}{|c|}{ Pneumonitis rates } & Overall survival & Comments \\
\hline $\begin{array}{l}\text { Bradley et al., } 2015 \\
\text { (RTOG 0617) (1) }\end{array}$ & 464 & IIB-IIIB & $4-7$ & & $28.7 \mathrm{mo}$ & $\begin{array}{l}\text { Benchmark study; all } \\
\text { had 3DCRT or IMRT }\end{array}$ \\
\hline Liao et al., 2017 (34) & 147 & IIB-IIIB, IV* & 6.5 & 10.5 (PSPT) & $28.8 \mathrm{mo}$ (29.5 mo IMRT, 26.1 mo PSPT) & Pneumonitis $P=0.15$ \\
\hline Chang et al., 2011 (35) & 44 & IIB-IIIB & & 2.3 (PSPT) & $29.4 \mathrm{mo}$ & \\
\hline Hoppe et al., 2016 (37) & 14 & & & 0 & $57 \%$ at $2 \mathrm{yr}$ & All had PBT \\
\hline Nguyen et al., 2015 (38) & 134 & IIB-IIIB & & 3 (PSPT) & $30.4 \mathrm{mo}(41-52.3 \%$ at $3 \mathrm{yr})$ & \\
\hline Tang et al., 2015 (39) & 341 & & 13 & 6 (PSPT) & & \\
\hline Ho et al., 2015 (40) & 66 & & & 7 (IMPT) & & \\
\hline Higgins et al., 2017 (43) & 348 & & & & 18.6 mo proton; 14 mo photon & \\
\hline
\end{tabular}

*, included patients with brain oligometastases, with recurrence after surgical resection, or with disease progression after chemotherapy. 3DCRT, 3-dimensional conformal (photon) radiation therapy; IMRT, intensity-modulated (photon) radiation therapy; PSPT, passively scattering proton therapy; IMPT, intensity-modulated proton therapy; PORT, postoperative radiation therapy.

escalated or the treatment to be accelerated, which can lead to better local control and reduced toxicity, particularly for patients with centrally located or bulky early-stage NSCLC who are not good candidates for SABR.

\section{Locally advanced NSCLC}

In contrast to early-stage disease, locally advanced NSCLC has a very high cancer-specific mortality rate and significant challenges for effective treatment. Radiotherapy remains the mainstay of treatment for disease at this stage, and the current standard of care includes chemotherapy in addition to radiation. Although locally advanced NSCLC is quite likely to metastasize, many patients with locally advanced disease die of the consequences of uncontrolled intrathoracic tumors, and hence therapies that improve local control are valuable in terms of survival.

Proton therapy has been evaluated for possible clinical advantages in terms of both toxicity and survival (Table 2) (1,34-43). Several retrospective studies have shown that proton therapy, compared with photon therapy, is associated with reduced lung, esophageal, and hematologic toxicity after concurrent chemoradiation, with acceptable rates of tumor control and survival (44). A prospective longitudinal observational study of 82 patients with unresectable primary or recurrent NSCLC treated with 3-dimensional conformal radiation therapy (3DCRT), IMRT, or proton therapy included patient-reported symptom burden, assessed weekly for up to 12 weeks with the validated MD Anderson Symptom Inventory. Fatigue was the most severe symptom over time regardless of treatment modality. Despite the fact that the proton group received significantly higher target radiation doses than did the IMRT and 3DCRT groups $(\mathrm{P}<0.001)$, patients receiving proton therapy reported significantly less severe symptoms than did patients receiving IMRT or 3DCRT (45).

A recent report of long-term clinical outcomes for patients with locally advanced NSCLC prospectively treated with concurrent proton therapy [60-74 Gy (RBE)] and chemotherapy in a nonrandomized case-only observational study showed excellent overall survival times of 40.4 months for patients with stage II disease and 30.4 months for patients with stage III disease, with acceptable toxicity (38). Consistent with these findings is the excellent median overall survival time of 29.4 months reported by Chang et al. from a single-arm phase II prospective study of concurrent chemotherapy and proton therapy to 74 GY (RBE) (35). A National Cancer Data Base analysis of patients treated with photon or proton therapy for NSCLC revealed that of more than 243,800 identified patients, only 348 had been treated with proton therapy. Despite the imbalance in patient numbers between the two treatment 
groups, propensity-matched analysis showed that proton therapy was associated with better survival (43).

Liao and colleagues reported findings from the first randomized trial to directly compare outcomes after PSPT versus IMRT, both with concurrent chemotherapy, for inoperable NSCLC (NCT00915005) (34). The hypothesis was that PSPT exposes less lung tissue to radiation than IMRT, thereby reducing toxicity without compromising tumor control. The primary endpoints were radiation pneumonitis and local failure. Eligible patients had stages IIB-IV NSCLC (patients with stage IV disease with a single brain metastasis were eligible, as were patients with recurrent lung or mediastinal disease after surgery) and were candidates for concurrent chemoradiation therapy. Pairs of treatment plans (for IMRT and PSPT) were created for each patient. Patients were eligible for randomization only if both plans satisfied the same prespecified dose-volume constraints for organs at risk at the same tumor dose. The results of this trial showed that PSPT did not improve dosevolume indices for lung or esophagus but did for heart. No benefit was noted in terms of pneumonitis or local failure rates after PSPT relative to IMRT. Compared with IMRT (given to 92 patients), PSPT (given to 57 patients) exposed less lung to doses of 5-10 Gy (RBE); more lung to $\geq 20 \mathrm{GY}$ (RBE); and less heart at all dose levels measured [5-80 GY (RBE)]. The radiation pneumonitis rate for all patients was 8.1\% (6.5\% IMRT, $10.5 \%$ PSPT), and the corresponding local failure rates were $10.7 \%$ (10.9\% and $10.5 \%$ ) (34).

Exploratory studies have suggested that PSPT leads to higher uptake of fluorodeoxyglucose by normal lung on positron emission tomography than does IMRT (46). Mean lung dose was found to be the only predictor of lung injury after IMRT, but the lung volume receiving high-dose radiation was the only predictor of lung injury after PSPT (47). Possible explanations for these findings may be the $3 \mathrm{D}$ conformal nature of PSPT and its associated planning and delivery processes, and the ability of IMRT to skirt normal critical structures, and the enlargement of the irradiated volume to accommodate the greater sensitivity of protons to inter- and intra-fractional uncertainties. These findings of a significant trade-off between proton conformity and use of large margins to account for the uncertainties in PSPT clinical delivery would theoretically be addressed by improving proton delivery and using IMPT technology.

RTOG 0617 identified heart V5 and V35 as predictors of overall survival. $\mathrm{Xu}$ and colleagues used a super-sensitive technique to measure cardiac troponin, a biomarker of cardiac injury, after chemoradiation. They found that cardiac troponin levels increased during thoracic radiation when the mean heart dose was 20 Gy or higher but did not change if the mean heart dose was 2 Gy or less. An increase of more than two-fold in troponin level relative to baseline (pretreatment) level was a significant predictor of poor overall survival (48). Thus far, all of the dosimetry studies comparing treatment plans for proton versus photon therapy have shown significant reductions of exposed heart volume at all dose levels. A secondary analysis of randomized trial NCT00915005 also showed significantly lower heart dose and reduced dose bath from PSPT (49). This reduction in unwanted irradiation of the heart may eventually translate to a survival benefit. Findings from RTOG 1308, a phase III randomized trial of protons and photons in which survival is the primary endpoint, will help to answer this question (50).

Lymphocytes, especially CD8 $\mathrm{T}$ cells, are a critical component of the antitumor immune response elicited by radiation therapy $(51,52)$. In 2014, Tang and others reported that the number of the lymphocytes begins to decline as early as the first fraction of radiation treatment for NSCLC, continues through the end of the treatment, and begins to recover thereafter. The nadir lymphocyte count was found to correlate with gross tumor volume and low-dose bath (using lung V5 as a surrogate). Most interestingly, lymphocyte nadir was found to correlate strongly with both progressionfree survival and overall survival (53). This finding has been confirmed in studies of other types of cancer, including esophageal cancer, liver cancer, and small cell lung cancer, suggesting that lymphopenia may be a common factor influencing overall survival across disease sites (54-58). Proton therapy can significantly decrease the low-dose bath for tumors at any location, and thus may be effective for avoiding lymphopenia. Active research is ongoing to understand the potential role of proton therapy in this regard.

Despite the standard approach of delivering radiosensitizing chemotherapy concurrently with radiotherapy for NSCLC, up to $40 \%$ of patients with have locoregional recurrence, of whom approximately one in four have an isolated locoregional recurrence (59). The management of recurrent NSCLC in patients who have had prior radiation therapy is particularly challenging. Historically, such patients have typically been treated with systemic therapy, either cytotoxic chemotherapy or, increasingly, immunotherapy, because of concerns that reirradiation may have significant and potentially fatal complications. Unfortunately, however, chemotherapy alone is not a curative approach and generally response rates are limited when it is used for recurrent disease (60). 
Particle therapy is ideally suited to the challenge of reirradiation (61). With the rapid dose falloff of radiation beyond the Bragg peak, particle therapy can most effectively spare critical organs at risk that may have already received high irradiation doses from prior treatments, thereby offering a potentially definitive treatment option with fewer risks of toxicity than photon therapy (62).

An early report on reirradiation with particle therapy was published by investigators from MD Anderson Cancer Center. Among 33 patients with intrathoracic recurrence of NSCLC, reirradiation with proton therapy (median dose $66 \mathrm{~Gy}$, at a median interval of 36 months after an initial course of $63 \mathrm{~Gy}$ ) led to an overall survival rate of $47 \%$ and a locoregional control rate of $54 \%$ at 1 year. Rates of severe (grade $\geq 3$ ) toxicity were $9 \%$ for esophageal toxicity and $21 \%$ for pulmonary toxicity (63). More recently, investigators from the same institution reported on the outcomes of 27 patients (22 of whom with NSCLC) treated with reirradiation with IMPT (median dose of 66 EQD2 Gy); at a median follow-up time of 11.2 months, the median overall survival time was 18.0 months, and the 1 -year freedom from local failure rate was 78\%. Doses of $66 \mathrm{EQD} 2 \mathrm{~Gy}$ or higher were associated with improved 1-year freedom from local failure ( $100 \%$ vs. $49 \%, \mathrm{P}=0.013)$. Toxicity was limited, with a $7 \%$ rate of late grade 3 pulmonary toxicity and no grade $\geq 3$ esophagitis (64).

Finally, a recent multicenter prospective study reported outcomes for 57 patients re-irradiated for locoregionally recurrent NSCLC (median dose 66.6 Gy). Two-thirds of those patients also received concurrent chemotherapy. At a median follow-up time of 7.8 months, the locoregional recurrence rate was $25 \%$, including $16 \%$ with a local recurrence. The 1-year overall survival rate was $59 \%$, and the 1-year progression-free survival rate was $58 \%$, and overall survival was found to decline as mean esophageal doses increased. Twenty-four patients ( $42 \%$ ) experienced grade $\geq 3$ acute or late toxicity; toxicity was more common when the tumor volume overlapped with the central airway region, and the mean esophageal and heart doses and use of concurrent chemotherapy were found to be associated with higher rates of toxicity (65).

\section{Summary and future directions}

Particle therapy has tremendous potential for treating thoracic cancer because of its superior dose distribution. However, the challenges in translating the dosimetric advantages of proton therapy to clinical benefits for patients with thoracic tumors are only beginning to be appreciated, and the development of technology to deliver proton therapy lags at least 20 years behind the development of the photon delivery technology. First, the greater vulnerability of protons to inherent heterogeneities in the beam path, to tumor and organ motion, to anatomic changes during the treatment, and to other factors poses significant challenges for proton treatment planning and for accurate and precise dose delivery. To fully realize the potential of particle therapy for thoracic cancer, extensive improvements are needed in all aspects of the treatment process, from simulation, planning algorithms, and volumetric image guidance through to realtime tracking and treatment adaptation. Highly conformal dose distribution is both basic and essential for demonstrating a clinical advantage for proton therapy in terms of preventing radiation pneumonitis, because expanding margins to counteract uncertainties would negate the dosimetric advantages of protons. Second, the commonly used normal tissue complication probability models based on photon therapy are not appropriate for proton therapy, and thus particle therapy-specific predictive models are needed that incorporate not only proton dose-distribution characteristics but also variations in RBE. Third, the need continues for designing and conducting "smart" proton therapy trials to establish clinical evidence and patient selection criteria to make proton therapy a truly personalized form of treatment. Future comparative trials could focus on endpoints that are common across all disease sites, such as cardiac toxicity, low-dose bath, and lymphopenia, instead of comparing one modality with another for a particular type of disease. Finally, new approaches to enhancing the dosimetric and biological advantages of proton therapy to improve clinical outcomes will require active and creative investigation, particularly image-guided hypofractionated IMPT and combinations of hypofractionated proton therapy with immunotherapy.

\section{Acknowledgements}

The authors thank Christine Wogan of MD Anderson's Division of Radiation Oncology for editorial contributions. Funding: Supported in part by U19CA021239 and by NIH Core grant to University of Texas MD Anderson Cancer Center.

\section{Footnote}

Conflicts of Interest: The authors have no conflicts of interest to declare. 


\section{References}

1. Bradley JD, Paulus R, Komaki R, et al. Standard-dose versus high-dose conformal radiotherapy with concurrent and consolidation carboplatin plus paclitaxel with or without cetuximab for patients with stage IIIA or IIIB non-smallcell lung cancer (RTOG 0617): a randomised, two-by-two factorial phase 3 study. Lancet Oncol 2015;16:187-99.

2. Minsky BD, Pajak TF, Ginsberg RJ, et al. INT 0123 (Radiation Therapy Oncology Group 94-05) phase III trial of combined-modality therapy for esophageal cancer: high-dose versus standard-dose radiation therapy. J Clin Oncol 2002;20:1167-74.

3. Wu ES, Oduyebo T, Cobb LP, et al. Lymphopenia and its association with survival in patients with locally advanced cervical cancer. Gynecol Oncol 2016;140:76-82.

4. Campian JL, Sarai G, Ye X, et al. Association between severe treatment-related lymphopenia and progressionfree survival in patients with newly diagnosed squamous cell head and neck cancer. Head Neck 2014;36:1747-53.

5. Kou F, Lu Z, Li J, et al. Pretreatment lymphopenia is an easily detectable predictive and prognostic marker in patients with metastatic esophagus squamous cell carcinoma receiving first-line chemotherapy. Cancer Med 2016;5:778-86.

6. Wild AT, Ye X, Ellsworth SG, et al. The Association Between Chemoradiation-related Lymphopenia and Clinical Outcomes in Patients With Locally Advanced Pancreatic Adenocarcinoma. Am J Clin Oncol 2015;38:259-65.

7. Mohan R, Grosshans D. Proton therapy - Present and future. Adv Drug Deliv Rev 2017;109:26-44.

8. Durante M, Paganetti H. Nuclear physics in particle therapy: a review. Rep Prog Phys 2016;79:096702.

9. Zhang X, Li Y, Pan X, et al. Intensity-modulated proton therapy reduces the dose to normal tissue compared with intensity-modulated radiation therapy or passive scattering proton therapy and enables individualized radical radiotherapy for extensive stage IIIB non-small-cell lung cancer: a virtual clinical study. Int J Radiat Oncol Biol Phys 2010;77:357-66.

10. Ishikawa H, Hashimoto T, Moriwaki T, et al. Proton beam therapy combined with concurrent chemotherapy for esophageal cancer. Anticancer Res 2015;35:1757-62.

11. Paganetti H. Relative biological effectiveness (RBE) values for proton beam therapy. Variations as a function of biological endpoint, dose, and linear energy transfer. Phys Med Biol 2014;59:R419-72.
12. Gerweck LE, Kozin SV. Relative biological effectiveness of proton beams in clinical therapy. Radiother Oncol 1999;50:135-42.

13. Britten RA, Nazaryan V, Davis LK, et al. Variations in the RBE for cell killing along the depth-dose profile of a modulated proton therapy beam. Radiat Res 2013;179:21-8.

14. Guan F, Bronk L, Titt U, et al. Spatial mapping of the biologic effectiveness of scanned particle beams: towards biologically optimized particle therapy. Sci Rep 2015;5:9850.

15. Moyers MF. Proton therapy. In: VanDyk J. editor. The Modern Technology of Radiation Oncology. A Compendium for Medical Physicists and Radiation Oncologists. Madison, WI: Medical Physics, 1991.

16. Hui Z, Zhang X, Starkschall G, et al. Effects of interfractional motion and anatomic changes on proton therapy dose distribution in lung cancer. Int J Radiat Oncol Biol Phys 2008;72:1385-95.

17. Liu W, Zhang X, Li Y, et al. Robust optimization of intensity modulated proton therapy. Med Phys 2012;39:1079-91.

18. Liu W, Liao Z, Schild SE, et al. Impact of respiratory motion on worst-case scenario optimized intensity modulated proton therapy for lung cancers. Pract Radiat Oncol 2015;5:e77-86.

19. Iwata H, Murakami M, Demizu Y, et al. High-dose proton therapy and carbon-ion therapy for stage I nonsmall cell lung cancer. Cancer 2010;116:2476-85.

20. Nakayama H, Sugahara S, Tokita M, et al. Proton beam therapy for patients with medically inoperable stage I nonsmall-cell lung cancer at the University of Tsukuba. Int J Radiat Oncol Biol Phys 2010;78:467-71.

21. Bush DA, Cheek G, Zaheer S, et al. High-dose hypofractionated proton beam radiation therapy is safe and effective for central and peripheral early-stage non-small cell lung cancer: results of a 12-year experience at Loma Linda University Medical Center. Int J Radiat Oncol Biol Phys 2013;86:964-8.

22. Iwata H, Demizu Y, Fujii O, et al. Long-term outcome of proton therapy and carbon-ion therapy for large (T2aT2bN0M0) non-small-cell lung cancer. J Thorac Oncol 2013;8:726-35.

23. Fujii O, Demizu Y, Hashimoto N, et al. A retrospective comparison of proton therapy and carbon ion therapy for stage I non-small cell lung cancer. Radiother Oncol 2013;109:32-7.

24. Kanemoto A, Okumura T, Ishikawa H, et al. Outcomes and prognostic factors for recurrence after high-dose 
proton beam therapy for centrally and peripherally located stage I non--small-cell lung cancer. Clin Lung Cancer 2014;15:e7-12.

25. Makita C, Nakamura T, Takada A, et al. High-dose proton beam therapy for stage I non-small cell lung cancer: Clinical outcomes and prognostic factors. Acta Oncologica 2015;54:307-14

26. Timmerman R, McGarry R, Yiannoutsos C, et al. Excessive toxicity when treating central tumors in a phase II study of stereotactic body radiation therapy for medically inoperable early-stage lung cancer. J Clin Oncol 2006;24:4833-9.

27. Wink KC, Roelof E, Simone CB 2nd, et al. Photons, protons or carbon ions for stage I non-small cell lung cancer - Results of the multicentric ROCOCO in silico study. Radiother Oncol 2018. [Epub ahead of print].

28. Chi A, Chen H, Wen S, et al. Comparison of particle beam therapy and stereotactic body radiotherapy for early stage non-small cell lung cancer: A systematic review and hypothesis-generating meta-analysis. Radiother Oncol 2017;123:346-54

29. Chang JY, Senan S, Paul MA, et al. Stereotactic ablative radiotherapy versus lobectomy for operable stage I nonsmall-cell lung cancer: a pooled analysis of two randomised trials. Lancet Oncol 2015;16:630-7.

30. Timmerman R, Paulus R, Galvin J, et al. Stereotactic body radiation therapy for inoperable early stage lung cancer. JAMA 2010;303:1070-6.

31. Onishi H, Araki T, Shirato H, et al. Stereotactic hypofractionated high-dose irradiation for stage I nonsmall cell lung carcinoma: clinical outcomes in 245 subjects in a Japanese multiinstitutional study. Cancer 2004;101:1623-31.

32. Stahl JM, Ross R, Harder EM, et al. The effect of biologically effective dose and radiation treatment schedule on overall survival in stage I non-small cell lung cancer patients treated With stereotactic body radiation therapy. Int J Radiat Oncol Biol Phys 2016;96:1011-20.

33. Koshy M, Malik R, Weichselbaum RR, et al. Increasing radiation therapy dose is associated with improved survival in patients undergoing stereotactic body radiation therapy for stage I non-small-cell lung cancer. Int J Radiat Oncol Biol Phys 2015;91:344-50.

34. Liao Z, Lee JJ, Komaki R, et al. Bayesian adaptive randomization trial of passive scattering proton therapy and intensity-modulated photon radiotherapy for locally advanced non-small-cell lung cancer. J Clin Oncol 2018:JCO2017740720.

35. Chang JY, Komaki R, Lu C, et al. Phase 2 study of high- dose proton therapy with concurrent chemotherapy for unresectable stage III nonsmall cell lung cancer. Cancer 2011;117:4707-13.

36. Hoppe BS, Flampouri S, Henderson RH, et al. Proton therapy with concurrent chemotherapy for non-small-cell lung cancer: technique and early results. Clin Lung Cancer 2012;13:352-8.

37. Hoppe BS, Henderson R, Pham D, et al. A phase 2 trial of concurrent chemotherapy and proton therapy for stage III non-small cell lung cancer: results and reflections following early closure of a single-institution study. Int J Radiat Oncol Biol Phys 2016;95:517-22.

38. Nguyen QN, Ly NB, Komaki R, et al. Long-term outcomes after proton therapy, with concurrent chemotherapy, for stage II-III inoperable non-small cell lung cancer. Radiother Oncol 2015;115:367-72.

39. Tang C, Gomez DR, Wang H, et al. Association between white blood cell count following radiation therapy with radiation pneumonitis in non-small cell lung cancer. Int J Radiat Oncol Biol Phys 2014;88:319-25.

40. Ho JC, Li H, Allen P, et al. Clinical Outcome of Intensity Modulated Proton Therapy for Non-Small Cell Lung Cancer. International Journal of Radiation Oncology Biology Physics 2015;93:188.

41. Remick JS, Schonewolf C, Gabriel P, et al. First clinical report of proton beam therapy for postoperative radiotherapy for non-small-cell lung cancer. Clin Lung Cancer 2017;18:364-71.

42. Harada H, Fuji H, Ono A, et al. Dose escalation study of proton beam therapy with concurrent chemotherapy for stage III non-small cell lung cancer. Cancer Sci 2016;107:1018-21.

43. Higgins KA, O'Connell K, Liu Y, et al. National Cancer Database analysis of proton versus photon radiation therapy in non-small cell lung cancer. Int J Radiat Oncol Biol Phys 2017;97:128-37.

44. Sejpal S, Komaki R, Tsao A, et al. Early findings on toxicity of proton beam therapy with concurrent chemotherapy for nonsmall cell lung cancer. Cancer 2011;117:3004-13.

45. Wang XS, Shi Q, Williams LA, et al. Prospective study of patient-reported symptom burden in patients with non-small-cell lung cancer undergoing proton or photon chemoradiation therapy. J Pain Symptom Manage 2016;51:832-8.

46. Yue J, McKeever M, Sio T, et al. Association of lung fluorodeoxyglucose uptake with radiation pneumonitis after concurrent chemoradiation for non-small cell lung cancer. Clin Transl Radiat Oncol 2017;4:1-7. 
47. Shusharina N, Liao Z, Mohan R, et al. Differences in lung injury after IMRT or proton therapy assessed by 18 FDG PET imaging. Int J Radiother Oncol Biol Phys 2018. [Epub ahead of print].

48. Xu T, Meng QH, Gomez DR, et al. Serum troponin T levels are associated with radiation dose to heart during definitive chemoradiation therapy for non-small cell lung cancer (abstract). Int J Radiat Oncol Biol Phys 2015;93:E411-2.

49. Deist T, Yang P, Oberije C, et al. Dosimetric analysis of randomized lung proton and photon plans with respect to radiation toxicity (OC-1144). Radiother Oncol 2017;123:S70-1.

50. Giaddui T, Chen W, Yu J, et al. Establishing the feasibility of the dosimetric compliance criteria of RTOG 1308: phase III randomized trial comparing overall survival after photon versus proton radiochemotherapy for inoperable stage II-IIIB NSCLC. Radiat Oncol 2016;11:66.

51. Formenti SC, Demaria S. Combining radiotherapy and cancer immunotherapy: a paradigm shift. J Natl Cancer Inst 2013;105:256-65.

52. Twyman-Saint Victor C, Rech AJ, Maity A, et al. Radiation and dual checkpoint blockade activate non-redundant immune mechanisms in cancer. Nature 2015;520:373-7.

53. Tang C, Liao Z, Gomez D, et al. Lymphopenia association with gross tumor volume and lung V5 and its effects on non-small cell lung cancer patient outcomes. Int J Radiat Oncol Biol Phys 2014;89:1084-91.

54. Feng JF, Liu JS, Huang Y. Lymphopenia predicts poor prognosis in patients with esophageal squamous cell carcinoma. Medicine (Baltimore) 2014;93:e257.

55. Grossman SA, Ellsworth S, Campian J, et al. Survival in patients with severe lymphopenia following treatment with radiation and chemotherapy for newly diagnosed solid tumors. J Natl Compr Canc Netw 2015;13:1225-31.

Cite this article as: Liao Z, Simone CB 2nd. Particle therapy in non-small cell lung cancer. Transl Lung Cancer Res 2018;7(2):141-152. doi: 10.21037/tlcr.2018.04.11
56. Mendez JS, Govindan A, Leong J, et al. Association between treatment-related lymphopenia and overall survival in elderly patients with newly diagnosed glioblastoma. J Neurooncol 2016;127:329-35.

57. Cho O, Oh YT, Chun M, et al. Radiation-related lymphopenia as a new prognostic factor in limited-stage small cell lung cancer. Tumour Biol 2016;37:971-8.

58. Kuo P, Bratman SV, Shultz DB, et al. Galectin-1 mediates radiation-related lymphopenia and attenuates NSCLC radiation response. Clin Cancer Res 2014;20:5558-69.

59. Curran WJ, Paulus R, Langer CJ, et al. Sequential vs. concurrent chemoradiation for stage III non-small cell lung cancer: randomized phase III trial RTOG 9410. J Natl Cancer Inst 2011;103:1452-60.

60. Noble J, Ellis PM, Mackay JA, et al. Second-line or subsequent systemic therapy for recurrent or progressive non-small cell lung cancer: a systematic review and practice guideline. J Thorac Oncol 2006;1:1042-58.

61. Simone CB 2nd, Rengan R. The use of proton therapy in the treatment of lung cancers. Cancer J 2014;20:427-32.

62. Lin SH, Merrell KW, Shen J, et al. Multi-institutional analysis of radiation modality use and postoperative outcomes of neoadjuvant chemoradiation for esophageal cancer. Radiother Oncol 2017;123:376-81.

63. McAvoy SA, Ciura KT, Rineer JM, et al. Feasibility of proton beam therapy for reirradiation of locoregionally recurrent non-small cell lung cancer. Pract Radiat Oncol 2018;8:58-65.

64. Ho JC, Nguyen QN, Li H, et al. Reirradiation of thoracic cancers with intensity modulated proton therapy. Pract Radiat Oncol 2017;in press.

65. Chao HH, Berman AT, Simone CB 2nd, et al. Multiinstitutional prospective study of reirradiation with proton beam radiotherapy for locoregionally recurrent non-small cell lung cancer. J Thorac Oncol 2017;12:281-92. 